\title{
Chemistry in Protoplanetary Nebulae
}

\author{
D A HOWE, T J MILLAR and D A WILLIAMS \\ Department of Mathematics, UMIST, PO Box 88, Manchester M60 1QD, UK
}

September 12, 1991

\begin{abstract}
We have investigated gas-phase chemistry in a remnant red giant wind, during transition to a planetary nebula, using the interacting stellar winds model. Rapid destruction by UV of most existing molecules is predicted, within $\sim 100 \mathrm{yrs}$ of the core star heating up, suggesting that the large molecules in CRL 618 may be destroyed within decades. However, significant abundances of some hydrogenated molecules and ions (eg. $\left.\mathrm{CH}^{+}, \mathrm{CH}_{2}^{+}, \mathrm{CH}_{3}^{+}, \mathrm{CH}, \mathrm{CH}_{2}, \mathrm{NH}\right)$ may form behind the shock predicted by the interacting stellar winds model. Also, survival and/or formation of observable amounts of some molecules (eg. $\mathrm{HCN}, \mathrm{CN}, \mathrm{HC}_{3} \mathrm{~N}$ ) may occur in dense clumps which survive transition, and may explain the existence eg. of HCN in NGC 7027.
\end{abstract}

\section{Introduction}

Planetary nebulae (PNe) are thought to form from winds of red giant ( $\mathrm{RG}$ ) stars, either as a result of sudden envelope ejection, or after steady envelope depletion during a period of high mass loss. A model has been developed (eg. by Kwok, 1983; Kahn, 1983), which assumes the latter case. This model, called the interacting stellar winds (ISW) model, involves an expanding bubble of ionised gas surrounding the hot, exposed stellar core. This bubble pushes against the remnant, high density "superwind", and progressively sweeps it into a thin, dense shell, with a shock at its leading edge. Meanwhile, the UV from the central star causes some ionisation and dissociation. We have constructed models to investigate the origin and fate of observed molecules and to predict others which might be observable in transition objects.

\section{Destruction of molecules in the precursor wind}

High mass loss RG winds (eg. IRC+10216) show a variety of molecular species, evidence of a rich chemistry. Our models predict that, during transition to $\mathrm{PN}$, most existing molecules (except $\mathrm{H}_{2}$ and possibly $\mathrm{CO}$, which self-shield) will be destroyed, within about $100 \mathrm{yrs}$ of the star becoming hot.

\section{Chemistry in the shocked neutral wind}

We have constructed a chemistry appropriate for a carbon-rich object. We find that significant amounts (column densities $\sim 10^{12}-10^{14} \mathrm{~cm}^{-2}$ ) of $\mathrm{CH}^{+}$, $\mathrm{CH}_{2}^{+}, \mathrm{CH}_{3}^{+}, \mathrm{CH}, \mathrm{CH}_{2}$ and $\mathrm{NH}$ may be sustained for the first few hundred years. 


\section{Chemistry in a dense clump}

Dyson et al. (1989) suggest that dense clumps in the Helix nebula (NGC 7293) may have originated as $\mathrm{SiO}$ maser spots in the wind of its RG precursor, and have survived transition to PN. We find that the UV extinction inside such a clump may allow the survival and/or formation of small but observable amounts of some molecules (eg. $\mathrm{HCN}, \mathrm{CN}, \mathrm{HC}_{3} \mathrm{~N}$ in a carbon-rich nebula) well into PN evolution.

\section{Discussion and conclusions}

Some proposed proto-PNe, eg. CRL 2688 and CRL 618 have large abundances of heavy molecules (eg. $\mathrm{HC}_{5} \mathrm{~N}, \mathrm{HC}_{7} \mathrm{~N}$ ), whereas most $\mathrm{PNe}$ have few or no molecules. Our results suggest that the large molecules formed in the RG wind will be destroyed by the UV flux from the exposed stellar core, within about $100 \mathrm{yrs}$ of the beginning of transition (assuming the star becomes hot before this). CRL 618 is thought to have ceased producing its "superwind" about 200 years ago, and its central star now has a temperature of about $25000 \mathrm{~K}$, so we expect that if the large molecules observed in this object are relics of the RG era, they will be destroyed within decades (we have not included molecular sources such as grain shattering, see Jura and Kroto, 1990).

Significant abundances of some hydrogenated molecules and molecular ions may be formed behind the shock predicted by the interacting stellar winds model, at least during the first few hundred years.

Survival and/or formation of small amounts of some molecules may occur in dense clumps, and may explain eg. the existence of HCN in the young PN, NGC 7027 (see eg. Sopka et al., 1989).

The above results will be presented in detail in Monthly Notices.

\section{References}

DYSON, J E, HARTQUIST, T W, PETTINI, M and SMITH, L J: 1989, Mon. Not. $R$. astr. Soc. 241, 625-630

JURA, M and KROTO, H: 1990, Astrophys. J. 351, 222-229

KAHN, F D: 1983, in Flower, D R, ed(s)., Planetary Nebulae, IAU Symp.No.103, Reidel, 305-316

KWOK, S: 1983, in Flower, D R, ed(s)., Planetary Nebulae, IAU Symp.No.103, Reidel, 293-303

SOPKA, R J, OLOFSSON, H, JOHANSSON, L E B, RIEU, N-Q, ZUCKERMAN, B: 1989, Astr. Astrophys. 210, 78-92 